\title{
Three-dimensional printing in structural heart disease and intervention
}

\author{
Yiting Fan ${ }^{1}$, Randolph H. L. Wong ${ }^{2}$, Alex Pui-Wai Lee ${ }^{1}$ \\ ${ }^{1}$ Division of Cardiology, Department of Medicine and Therapeutics, ${ }^{2}$ Division of Cardiothoracic Surgery, Prince of Wales Hospital, Faculty of \\ Medicine, The Chinese University of Hong Kong, Hong Kong, China \\ Contributions: (I) Conception and design: Y Fan, AP Lee; (II) Administrative support: AP Lee, RH Wong; (III) Provision of study materials or \\ patients: Y Fan; AP Lee; (IV) Collection and assembly of data: Y Fan; (V) Data analysis and interpretation: Y Fan; (VI) Manuscript writing: All \\ authors; (VII) Final approval of manuscript: All authors. \\ Correspondence to: Alex Pui-Wai Lee, MD, FRCP, FACC, FESC. Rm 114037, 9/F, Lui Che Woo Clinical Sciences Building, Prince of Wales Hospital, \\ Shatin, N.T., Hong Kong, China. Email: alexpwlee@cuhk.edu.hk.
}

\begin{abstract}
Three-dimensional (3D) printing refers to the process by which physical objects are built by depositing materials in layers based on a specific digital design. It was initially used in manufacture industry. Inspired by the technology, clinicians have recently attempted to integrate $3 \mathrm{D}$ printing into medical applications. One of the medical specialties that has recently made such attempt is cardiology, especially in the field of structural heart disease (SHD). SHD refers to a group of non-coronary cardiovascular disorders and related interventions. Obvious examples are aortic stenosis, mitral regurgitation, atrial septal defect, and known or potential left atrial appendage (LAA) clots. In the last decade, cardiologists have witnessed a dramatic increase in the types and complexity of catheter-based interventions for SHD. Current imaging modalities have important limitations in accurate delineation of cardiac anatomies necessary for SHD interventions. Application of 3D printing in SHD interventional planning enables tangible appreciation of cardiac anatomy and allows in vitro interventional device testing. 3D printing is used in diagnostic workup, guidance of treatment strategies, and procedural simulation, facilitating hemodynamic research, enhancing interventional training, and promoting patient-clinician communication. In this review, we attempt to define the concept, technique, and work flow of 3D printing in SHD and its interventions, highlighting the reported clinical benefits and unsolved issues, as well as exploring future developments in this field.
\end{abstract}

Keywords: Three-dimensional printing (3D printing); structural heart disease (SHD); cardiovascular intervention; cardiovascular imaging

Submitted Aug 31, 2019. Accepted for publication Sep 11, 2019.

doi: $10.21037 /$ atm.2019.09.73

View this article at: http://dx.doi.org/10.21037/atm.2019.09.73

\section{Introduction}

Three-dimensional (3D) printing, also known as rapid prototyping, additive manufacturing, or solid freeform technology, refers to the process by which physical objects are built by depositing materials in layers based on a specific digital design (1). This technology was first introduced by Jean Claude André, who subsequent abandoned the patent application, and Charles Hull in 1984, who coined the term "stereolithography" (SLA) and later co-founded 3D System Corporation. It was initially used in manufacture industry to create $3 \mathrm{D}$ models from digital images to allow users testing on a design before investment into larger scale manufacturing (2). Freedom of design, mass customization, waste minimization, and the ability to manufacture complex structures are the main advantages of SLA as a technology of manufacturing, which have made it wide adopted by various crafts and design industries (3). Inspired by the technology, clinicians have recently attempted to integrate $3 \mathrm{D}$ printing into medical applications, taking advantage of its merits of advanced visualization and procedural simulation to improve 
clinical practice. Dental, maxillofacial, and orthopaedic surgeons were among the first to pioneer the use of medical $3 \mathrm{D}$ printing. Cumulative experience from these specialties has encouraged cardiologists and other clinicians to enter the field.

Structural heart disease (SHD) refers to a group of non-coronary cardiovascular disorders and related interventions. Obvious examples are aortic stenosis, mitral regurgitation (MR), atrial septal defect (ASD), and known or potential left atrial appendage (LAA) clots. In the last decade, cardiologists have witnessed a dramatic increase in the types and complexity of catheter-based interventions for SHD. Compared to cardiac surgeons, interventional cardiologists cannot directly visualize or palpate the anatomy during catheter-based intervention, and rely heavily on imaging to understand complex anatomy (4). Unfortunately, current imaging modalities have important limitations in accurate delineation of cardiac anatomies. 2D-imaging, for instance, falls short both in representation of the complicated 3D relationships as well as assistance of corrective procedures (5). The physicians have to mentally reconstruct $2 \mathrm{D}$ images in different imaging planes to appreciate complex 3D spatial relationships (6). Although advances in $3 \mathrm{D}$ imaging enable display of $3 \mathrm{D}$ images, it is still only displayed on flat computer screens. Application of $3 \mathrm{D}$ printing in SHD interventional planning enables tangible appreciation of cardiac anatomy and allows in vitro interventional device testing (7). Application of 3D printing in SHD may answers to the pressing need for understanding the pathology and optimization of cardiac procedures for individual patients.

In SHD, 3D printing is used in diagnostic workup, guidance of treatment strategies $(7,8)$, and procedural simulation (9-11), facilitating hemodynamic research $(12,13)$, enhancing interventional training, and promoting patient-clinician communication $(14,15)$. Herein, we present a contemporary review to define the concept, technique, and work flow of medical 3D printing, highlighting the reported clinical benefits and unsolved issues, as well as exploring future developments in this field.

\section{Cardiovascular image data acquisition}

The protocol of $3 \mathrm{D}$ printing generally involves 3 steps: image acquisition, data post-processing, and manufacturing (Figure 1). Typical data source for medical 3D printing are volumetric medical image data sets (16). An imaging data set must be volumetric with high-spatial resolution and minimal artefact which allows non-ambiguous separation of the region of interest from surrounding structures/ blood pool. Moreover, dynamic datasets are preferred since they provide temporal resolution that allows selection of the optimal phase in cardiac cycle for printing (4). The most commonly used imaging sources for SHD are echocardiography $(17,18)$, computed tomography (CT) $(16,19)$ and magnetic resonance imaging (MRI) $(20,21)$. Other modalities, such as positron emission tomography, single photon emission CT and cone beam CT, are less commonly used (22). All images should use the common Digital Imaging and Communication in Medicine (DICOM) format $(23,24)$.

\section{$C T$}

The advantages of high spatial resolution, high image quality, and relatively easy image segmentation make CT the most commonly used modality for $3 \mathrm{D}$ printing (25-27). The use of CT contrast increases the signal-tonoise ratio of images, enabling convenient separation of the region of interest from surrounding structures during image segmentation. The timing of contrast injection is crucial for adequacy of $\mathrm{CT}$ images for $3 \mathrm{D}$ printing. Image segmentation may become difficult or non-feasible contrast opacification of structures is inadequate, or blooming artefacts created by metallic implants and/or calcifications are present. Cardiac CT has several limitations including radiation and nephrotoxic contrast exposure, and thus may not be the imaging modality of choice in paediatric patients or patients with renal insufficiency.

\section{D echocardiography (3DE)}

3DE, especially $3 \mathrm{D}$ transesophageal echo (TEE), is an attractive image data source for $3 \mathrm{D}$ printing. It is widely available, relatively low-cost, and does not require ionizing radiation (28). In mitral and tricuspid valve procedures, echocardiography rather than CT is often the primary imaging modality for preprocedural screening, intraprocedural monitoring, and postprocedural followup. $3 \mathrm{DE}$ has the highest temporal resolution among all $3 \mathrm{D}$ imaging modalities, allowing selection of images at the optimal timing in the cardiac cycle for 3D printing (29). Because contrast is generally not used during $3 \mathrm{DE}$, segmentation techniques for $3 \mathrm{DE}$ relies on contrast in echogenic intensity of different tissues against the blood pool. For tissue structures surrounded by blood pool, such 

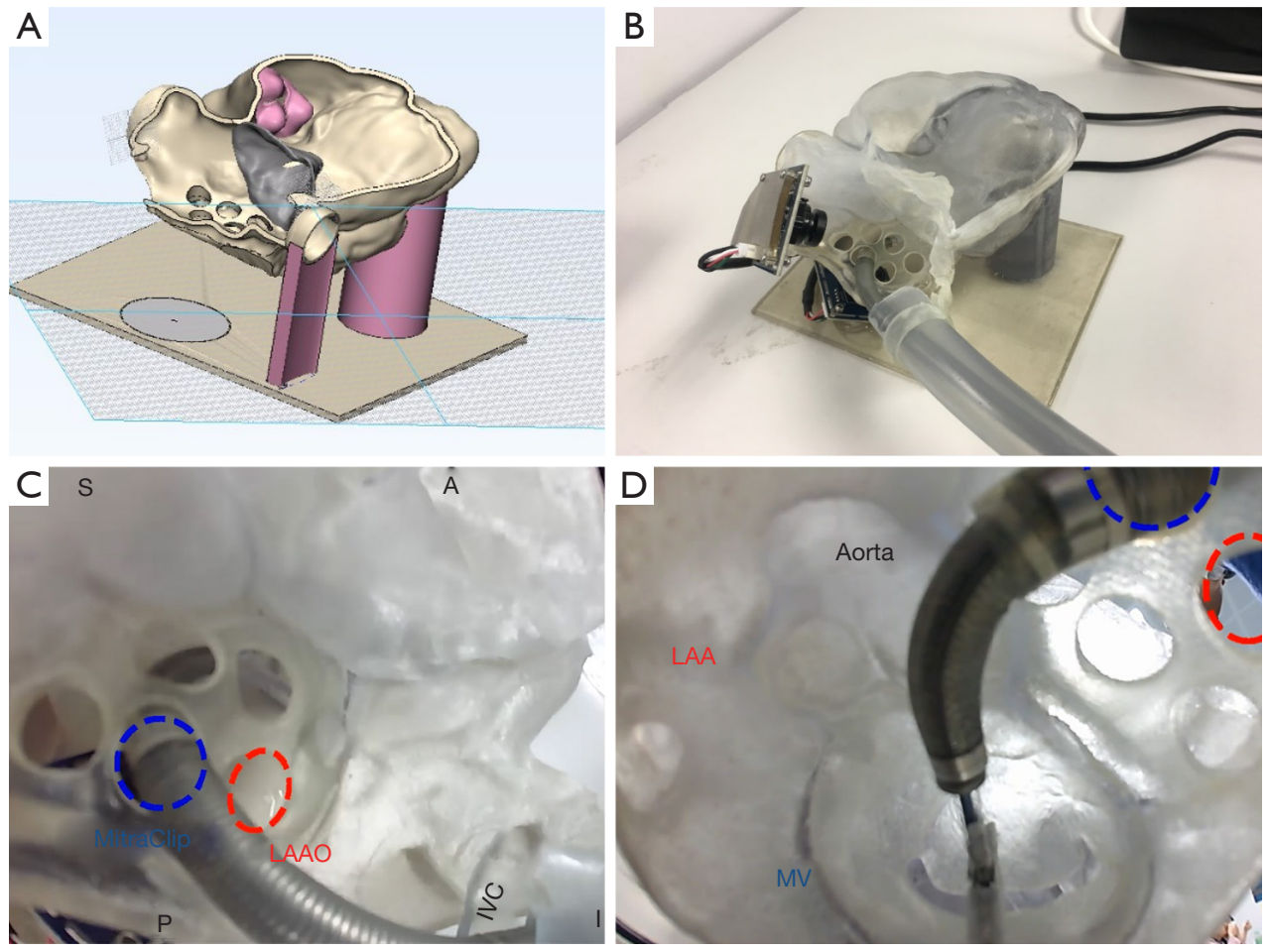

Figure 1 Pre-procedural simulation of MitraClip on 3D-printed model. (A) Digital model of a heart. Different colors stand for different cardiac components (grey: mitral valves; light pink: tricuspid valves; light gold: atrial septum, left atrium, left and right ventricle). (B) Multi-material 3D-printed heart model for pre-procedural simulation. The valves were printed with flexible material and the rests were printed with hard material. (C) The 5 holes drilled in the atrial septum represents the different position for different kinds of structural heart interventions. (D) The MiraClip device was released via delivery catheter through the atrial septum to the mitral valve. Blue circle: MitraClip; red circle: left atrial appendage occlusion (LAAO). S, superior; A, anterior; P, posterior; I, inferior; IVC, inferior vena cava; LAA, left atrial appendage; MV, mitral valve.

as valves and the atrial septum, direct thresholding to create a tissue mask is sufficient to create $3 \mathrm{D}$ models (17). For hollow structures such as the LAA, indirect segmentation of blood pool can separate the region of interest from the surroundings (30). Blood volume of the hollow structure is segmented first, and then a geometric surface mesh model of the hollow cast is refined to extract the internal structures.

There are limitations of $3 \mathrm{DE}$ as an imaging source for $3 \mathrm{D}$ printing. 3DE has a narrow field of view relative to $\mathrm{CT}$ or MRI (29). As a result, 3D models of the whole heart cannot be easily created in by $3 \mathrm{DE}$-based $3 \mathrm{D}$ printing. In case of LAA closure, for instance, 3D-TEE based 3D printing usually cannot include the atrial septum in the LAA model, hence the procedural step of transseptal puncture in LAA occlusion cannot be simulated. Furthermore, the signal-to-noise ratio of $3 \mathrm{DE}$ is relatively low compared to CT. More manual adjustments are needed during image segmentation, which may lead to more error and lower reproducibility, especially for fine structural details in the far field of 3DE images.

\section{Cardiac MRI}

Compared to 3DE and CT, cardiac MRI is of limited use for 3D printing. First, a full MRI scanning often takes over an hour to complete. During the scanning, patients are required to lie still and follow breath-holding commands intermittently. Therefore, it poses challenge to complete a cardiac MRI scan for patients with poor compliance, such as elderly and those with frailty and comorbidities (4). Sometimes an additional usage of sedation is required. Moreover, patients who have prior implantation of internal ferromagnetic devices are forbidden from entering the MRI machine. Second, similar to 3DE, MRI have a narrow field of view, thus may require specific scan protocols such as $3 \mathrm{D}$ 
full-phase navigated MRI when multiple structures are to be printed. Lastly, the slice thickness with minimal $3 \mathrm{~mm}$ of MRI is suboptimal, falling short in providing detailed replication in coronary arteries and valves; on the other hand, MRI is increasingly used in reconstruction of cardiac mass, congenital heart diseases and systemic vasculature disorders $(20,21,31)$.

\section{Post-processing of image data}

The second step in generation of a 3D-printed model is an image data post-processing procedure known as segmentation using dedicated image post-processing software (22). This process involves the automated or semi-automated delineation of the structures of interest and conversion to digital models of geometric meshwork. Segmentation requires comprehensive knowledge of the cardiac anatomy, image interpretation, and skills in using the image post-processing software (32). Several algorithms are available to perform image segmentation, the most frequently used being multi-planar reformation, region growing, surface/volume rendering, and maximal/minimal intensity projection (22).

During segmentation, the contours of the region of interest are identified on multiple 2D image planes and computationally transformed into $3 \mathrm{D}$ triangular surface meshwork. These data are usually stored and exported in standard tessellation language (STL) format, which can be sent to $3 \mathrm{D}$ printers for printing. The digital models generated at this stage are usually anamorphic and rough, which have to be further refined and optimized using computer-aided design software for $3 \mathrm{D}$ printing. The most frequently used digital model refinement techniques include surface smoothing, trimming, hollowing, and patching. Apart from being used for creation of $3 \mathrm{D}$ physical models, the digital models so generated can also be used for digital simulation of virtual procedures and device implantation on computer screen. The tissue-device interaction can be quantitively evaluated with the aid of analysis software (33-35).

\section{Creation of 3D-printed models}

The final step of $3 \mathrm{D}$ printing is fabrication of the physical models by $3 \mathrm{D}$ printers. Several printing techniques are available and the appropriate one(s) should be chosen according to the purpose of the application. In cardiovascular applications, widely used $3 \mathrm{D}$ printing techniques include fused deposition modelling (FDM), selective laser sintering (SLS), SLA, and material jetting (36). Other techniques such as injection molding, inkjet/ extrusion/laser-assisted bioprinting are seldom used in cardiovascular specialty.

Although various kinds of materials can be used in medical 3D printing, scant data exist for the mechanical properties of these materials compared with real cardiovascular tissues. Recently, some rubber-like 3D printing materials with capability of repeated flexing and bending are proved to have similar mechanical properties with cardiovascular tissues (37). Silicon has excellent resemblance to soft tissue; however, it is technically impossible to use silicon as printing material like rubbers or metals. The only feasible way of producing silicone models is injection molding (38). However, silicone is too soft and easily to be torn. It is now more commonly applied to the solid models or models with hard support. When it comes to the organs with big cavities but thin walls, such as atriums and vessels, the precise duplication is difficult to be realized.

Multi-material printing by material jetting is increasingly used to create cardiac structures. Different tissue components were printed with different textures. For instance, an aortic valve was printed with flexible printing material, and the calcifications attached to valves were printed with hard printing material, respectively.

\section{Applications of 3D printing in SHD interventions}

SHD refers to a wide range of non-coronary cardiac conditions and related interventions (39). The increasing complexity for SHD interventions and the limitations of current imaging modalities make 3D printing a potential game-changer in procedural planning. The application of 3D printing has several notable advantages. First, it helps to ease pre-procedural planning by providing anatomic prototype models of high accuracy. The $3 \mathrm{D}$-printed physical model can be hold on hands and inspected from full range of views, allowing excellent spatial appreciation of complex cardiovascular pathologies $(40,41)$. The tactile perception of models enables better understanding of anatomy and improved confidence in procedural planning, minimizing visual errors caused by 2D images (42). Second, in vitro procedural simulation on model provides guidance in catheter and device selection during the procedure. This may result in shortening of procedural time, reduction in device resizing, and decrease in radiation exposure (43). It also potentially helps to avoid procedural complications and 
improving procedural efficacy (44). Lastly, these life-sized models may reduce the learning curve of the interventional operators and enhance patient-clinician interactions (19) (Table 1).

\section{LAA occlusion}

Catheter-based LAA occlusion is an alternative to anticoagulation for stroke prevention in patients with nonvalvular atrial fibrillation (62). The complexity of LAA anatomy has been overlooked until recent development in LAA occlusion reminding us its heterogeneous and complex morphology. Planning of LAA occlusion usually uses peri-procedural 2D-TEE combining with fluoroscopy guidance and, less frequently, with pre-procedural CT. The unique and variable anatomy of the LAA poses a challenge for accurate sizing of the device. Reportedly $1.5 \%$ to $9.1 \%$ of cases fail to achieve implant success (63), and serious complications, in particular pericardial effusion, are reported in $4 \%$ of procedures $(62,64)$. Moreover, residual peri-device leak and device thrombus have been implicated in late thromboembolic events on long-term follow-up (65).

Successful implantation of the LAA device depends on accurate sizing of device landing zone and precise catheter positioning at the appropriate depth and orientation before device release (66). Patient-specific 3D-printed models allows detail assessment of the shape of the appendage, size of the ostium, angulation of the appendage body, and location of the trabeculations $(19,40,67)$. Besides, these models allow pre-procedural simulation of different sizes and types of devices, and can serve as guidance of device implantation strategy during the procedure. Device sizing guided by $3 \mathrm{D}$ printing is associated with improved procedural outcomes $(37,45)$. With the aid of software for $3 \mathrm{D}$ strain analysis, device-model interactions can be quantified in vitro (67). Potential complications such as peridevice leak and pericardial effusion resulting from incorrect sizing can be avoided. In addition to device selection, 3D printing allows fabrication of customized LAA devices as recently reported by Mosadegh's team (47). Their work offers an alternative solution in challenging cases with LAA morphologies incompatible with commercially available devices.

\section{Transcatheter aortic valve implantation (TAVI)}

TAVI has established as an alternative treatment for symptomatic severe aortic stenosis in candidates who are considered high risk for receiving traditional openheart surgery $(68,69)$. The procedure has not gained much attention until 2010s when positive results of the PARTNER trials were reported $(70,71)$. It is now generally accepted as a safe procedure preferred by patients, with its indications and patient volume expected to rapidly expand in near future. Procedural success in TAVI requires rigorous screening for suitable patients with a good understanding of how the transcatheter heart valve prosthesis, when deployed, would seat in the aortic root of the patients (72), especially in patients with complex aortic anatomy such as a porcelain aorta (73). 3D models allow direct visualization of valve pathology and hands-on simulation by physicians $(9,49)$. More importantly, pre-procedural simulation on $3 \mathrm{D}$ models can identify potential complications in complex cases (74) (Figure 2A,B,C).

In a case series reported by our group (75), procedural simulation on 3D-printed aortic root models enables preprocedural prediction and post-procedural investigation of the mechanisms of coronary occlusion, a rare but potentially fatal TAVI complication in patients with highrisk anatomy (i.e., low-lying coronary ostium, small aortic root, valve-in-valve implantation). In a patient reported by Lee et al. (76), procedural simulation on 3D model predicts and unveils the mechanism of coronary obstruction caused by displacement of the bulky calcified left cusp of the aortic valve by the self-expanding prosthesis towards the left coronary ostium. Moreover, simulations on patient-specific $3 \mathrm{D}$-printed models can predict the occurrence of postTAVI paravalvular leaks in the actual procedure $(50,51)$. Qian $e t$ al. demonstrated the feasibility of using 3D printed aortic root phantoms composed of tissue-mimicking metamaterials to quantify post-TAVI aortic root strain in vitro; the proposed annular bulge index achieved a high level of accuracy in predicting severity and locations of post-TAVI paravalvular leaks (50). In addition to anatomic information, fabricated patient-specific aortic valve models can be coupled to pulsatile flow loop hemodynamic simulators to assess the functional characteristics of the valve under various controlled forward flow conditions (52). Such simulation platforms are potentially useful for physiologic evaluation of the stenotic aortic valve and hemodynamic consequence of aortic valve intervention such as TAVI.

\section{Transcatheter mitral valve intervention (TRAMI)}

According to a population-based study $(77,78), M R$ is the most frequent valve disease with a prevalence of $9.3 \%$ in 
Table 1 Characteristics of applications of 3D printing in different cardiovascular scenarios

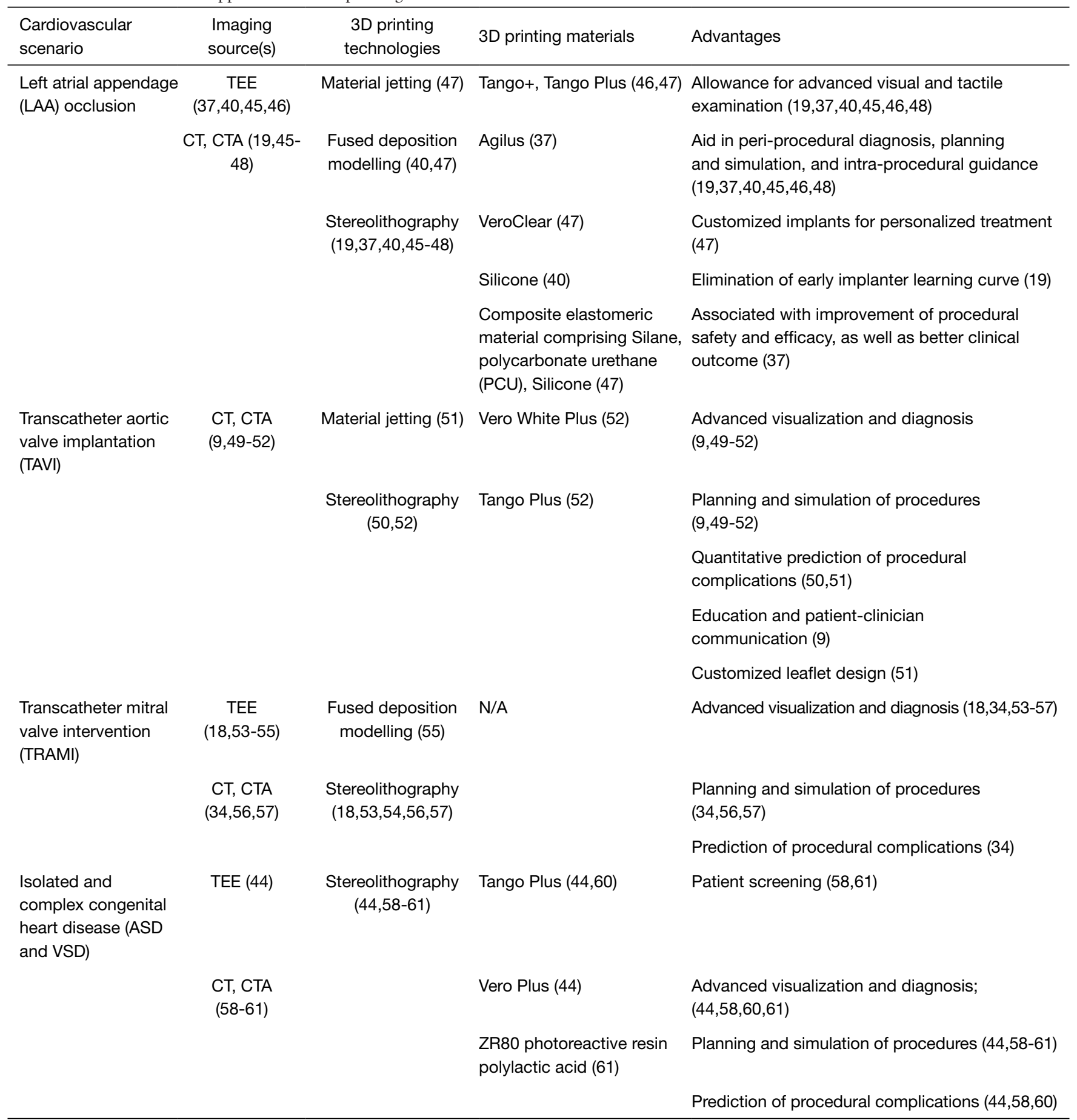

3D, three-dimensional; CT, computed tomography; CTA, computed tomography angiography; TEE, transesophageal echo; ASD, atrial septal defect; VSD, ventricular septal defect. 

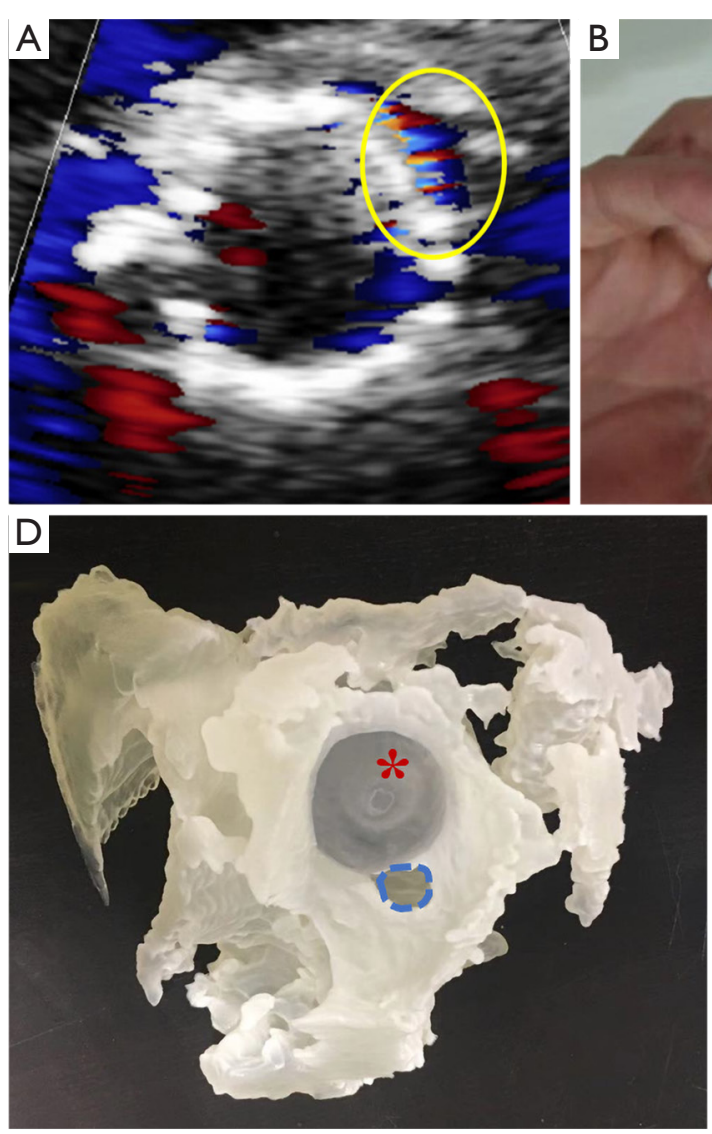
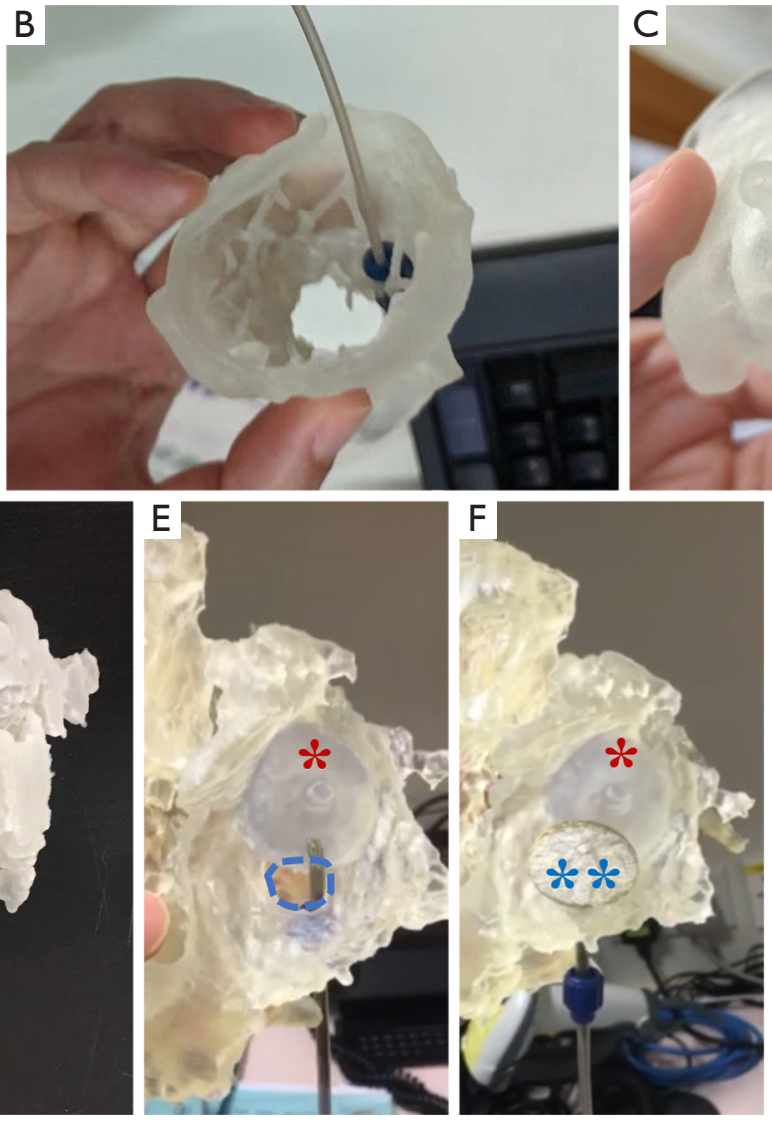
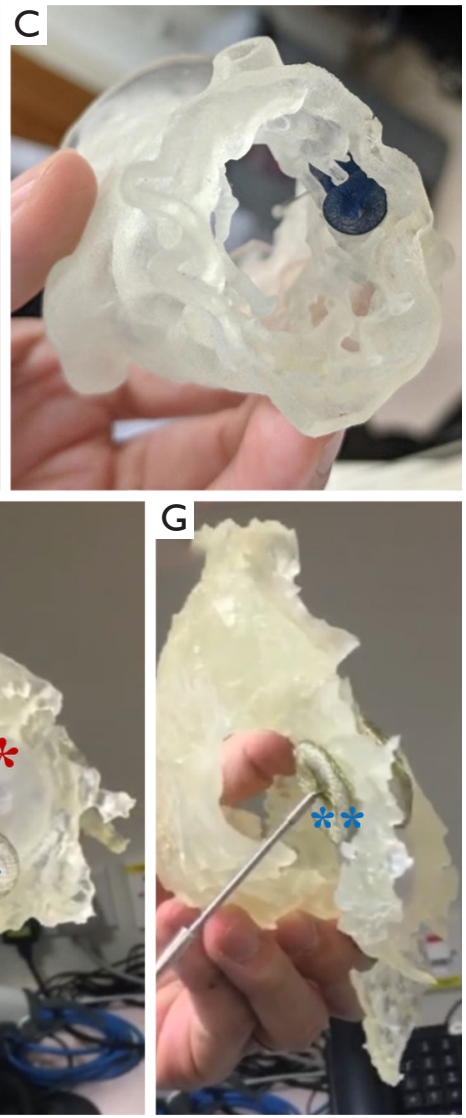

Figure 2 Application of 3D printing for peri-device leak. (A,B,C) A case found with peri-device leak post TAVI and needed peri-device leak occlusion: (A) routine TEE post-TAVI showed peri-device leak (yellow circle); (B) simulation of peri-device leak occlusion on 3D-printed aortic root model derived from post-TAVI CT; (C) the 10-mm vascular plug was found to be best-fit for this case. (D,E,F,G) A case found with residual leak after ASD closure: (D) multi-material 3D printed model showed residual leak (blue circle) next to the ASD occluder (asterisk *); (E) the delivery catheter went through the leak position; (F) the device (two asterisks **) was released in situ. (G) The bicaval view of 3D-printed model showed stable release and stay of the chosen device. 3D, three-dimensional; TAVI, transcatheter aortic valve implantation; TEE, transesophageal echo; CT, computed tomography; ASD, atrial septal defect.

patients $\geq 75$ years old. Until recently, for elderly patients or patient with co-morbidities who are deemed inoperable or high risk for conventional surgery, effective treatment option has been lacking. With the advances in transcatheter techniques and devices, less invasive TRAMI for MR has become available (72). There are currently two main TRAMI approaches. The first approach is transcatheter mitral valve repair. The most widely adopted transcatheter repair technique is the MitraClip ${ }^{\mathrm{TM}}$ system (Abbott; Santa Clara, CA, USA), which is a catheter-based edge-to-edge leaflet repair technique. During the MitraClip ${ }^{\mathrm{TM}}$ procedure, a clip-like device is deployed through a transfemoral transseptal approach to the mitral leaflets, recreating leaflet coaptation to achieve MR reduction. The worldwide volume of this procedure is constantly growing (79). The second TRAMI approach is transcatheter mitral valve replacement, in which a bioprosthetic valve is delivered in the mitral position to replace the diseased one (80). These less invasive percutaneous techniques are attractive treatment alternatives for severe MR in patients deemed high risk for conventional surgery.

As the anatomy of mitral valve is more complex than that of aortic valve, successful procedural planning for TRAMI requires even more individualized assessment than TAVI. The introduction of $3 \mathrm{D}$ printing in TRAMI has revolutionized how cardiologists understand mitral valve anatomy and predict the impact of various interventions on the mitral valve. It complements conventional imaging 
in patient and device selection. Effects of various TRAMI techniques on the mitral valve apparatus can be better appreciated through device simulation on tissue-mimicking $3 \mathrm{D}$ models (81) and complications not predicted by conventional imaging can potentially be revealed (82). $3 \mathrm{D}$ modelling may have a role in innovating personalized approach to treat complex MR lesions combing multiple different devices (56). 3D simulated models predict left ventricular outflow tract obstruction in patients undergoing transcatheter mitral valve replacement prompting anterior mitral leaflet resection and alcohol septal ablation prior to procedure (83).

\section{Congenital heart diseases}

Congenital heart diseases occur in approximately $1 \%$ of live births. ASD are among the most common form of congenital heart diseases. Therapeutic intervention of ASD has evolved from open heart surgery to percutaneous therapy with closure devices. Percutaneous closure of secundum ASD is highly feasible and safe. Imaging with echocardiography is used to guide these procedures with excellent long-term outcome $(84,85)$. Closure of multiple or fenestrated ASD, or complex residual peri-device defects, however, require more careful planning. 3D printing can help in precise location of the anatomic landmarks which may not be appreciated adequately on conventional imaging modalities, and facilitate selection of device size with preprocedural simulation. Primary imaging data sources for 3D printing in ASD and other congenital heart disease include CT (58), echocardiography (17), and cardiac MRI (86). 3D models provide vivid demonstration of the defects and their $3 \mathrm{D}$ relations with surrounding structures. Importantly, 3D model simulation aids to assess whether there are sufficient tissue rims for secure device anchor (58). In a patient with partial device prolapse after percutaneous ASD closure and recurrent left-to-right interatrial shunt around the rim of the first device, $3 \mathrm{D}$ printing parts with multiple materials proved to be advantageous by allowing realistic procedural planning on structures consisting of soft cardiac tissues and rigid implanted prosthesis (44) (Figure 2D,E,F,G).

\section{Unresolved issues and future direction}

Despite the enthusiasm in applying $3 \mathrm{D}$ printing cardiovascular medicine, there remains several issues that need to be resolved before widespread adoption of this technology. Current limitation of $3 \mathrm{D}$ printing includes the lack of validated technical standard, increased upfront costs of the technology, and scant evidence on the added clinical benefit.

The accuracy of replication of the cardiac structural geometry must be validated across different source imaging modalities, 3D printing methods, and cardiovascular modelling scenarios. Standardizations of the source image data acquisition protocol and image post-processing techniques are important. Although different imaging modalities are complementary and generally show good correlations in anatomic measurements, the results have been limited to small case series (87-89). Therefore, more head-to-head comparisons are needed to provide data for developing standard protocols. A streamlined workflow is needed so extra schedule for additional imaging acquisitions can be avoided before $3 \mathrm{D}$ printing (90).

There are different types of $3 \mathrm{D}$ printers that vary in printing techniques used, materials employed for printing, time-to-print, learning curve, cost, and finish quality of the product. The most frequently reported 3D printing technologies in cardiovascular medicine include FDM, SLS, SLA, and material jetting. The accuracy and reproducibility (intra- and inter-operator variability) of clinical 3D-printed models generated by different printing technologies need to be validated and compared before wide incorporation into clinical practice. Such comparisons are lacking particularly in the field of cardiovascular 3D printing in SHD interventions. The mechanical properties of the 3D-printed materials, such as tensile strength, elasticity, flexibility, hardness, and durability have utmost importance for cardiovascular applications (56). The majority of cardiovascular applications reported so far have employed materials with properties that have not been meticulously compared with the cardiovascular tissue they are mimicking. Validation of 3D-printed material properties against actual human patient tissues is important to ensure that procedural simulation is realistic.

Cost is a major barrier to widespread adoption of $3 \mathrm{D}$ printing. Most of the cost in $3 \mathrm{D}$ printing can be attributed to dedicated hardware and software, 3D printers, materials, and labour. The learning curve of $3 \mathrm{D}$ printing for clinical and engineering users remains a major challenge since interpretation of cardiac anatomy and pathology from different imaging source and image post-processing and operation of the $3 \mathrm{D}$ printing require both medical and engineering expertise. Communications between clinicians and engineers regarding the specific clinical demands and available technical solutions for individual cases remain the key to success in implementation of $3 \mathrm{D}$ printing in 
clinical care.

Last but not least, the added benefits of $3 \mathrm{D}$ printing in terms of improvement in clinically meaningful outcomes need to be systematically defined in clinical studies. There is a growing body of evidence linking the use of $3 \mathrm{D}$ printing as guidance of SHD intervention with improved procedural outcomes $(37,45,91)$. Randomized clinical trials are warranted to provide robust outcome data on the effect of $3 \mathrm{D}$ printing on procedural efficacy and safety, procedural time, patient-physician communication, as well as the costeffectiveness of the technology.

\section{Conclusions}

Cardiovascular $3 \mathrm{D}$ printing is a novel tool that has the potential to revolutionize the planning of SHD intervention. With the rapid increase in the availability and variety of percutaneous therapies and devices in SHD, it is imperative that interventional cardiologists are able to have a deep understanding of the spatial relationship of complex cardiovascular anatomy in SHD. The advent of $3 \mathrm{D}$ printing technology is able to create anatomically accurate, patient-specific physicals model, converting virtual 3D images on flat computer screen to palpable 3D models suited for interventional simulation. Patient-specific 3D-printed models of cardiovascular structures have emerged as promising tools for pre-procedural planning, intra-procedural decision making, and post-procedural evaluation of the mechanisms of procedure-related complications should they occur. Nevertheless, widespread clinical adoption of $3 \mathrm{D}$ printing in SHD interventions is currently limited owing to the lack of robust evidence that systematically demonstrates the effectiveness, technical barrier, and increased upfront cost. Further effort in technical standardization, and clinical evaluation of added benefit and cost-effectiveness of $3 \mathrm{D}$ printing are needed to bring this promising technique to clinical reality.

\section{Acknowledgments}

Funding: This work was funded by the Hong Kong Special Administrative Region Government Health and Medical Research Fund (No. 05160976).

\section{Footnote}

Conflicts of Interest: The authors have no conflicts of interest to declare.
Ethical Statement: The authors are accountable for all aspects of the work in ensuring that questions related to the accuracy or integrity of any part of the work are appropriately investigated and resolved.

\section{References}

1. Ventola CL. Medical Applications for 3D Printing: Current and Projected Uses. P T 2014;39:704-11.

2. Ligon SC, Liska R, Stampfl J, et al. Polymers for 3D Printing and Customized Additive Manufacturing. Chem Rev 2017;117:10212-90.

3. Tack P, Victor J, Gemmel P, et al. 3D-printing techniques in a medical setting: a systematic literature review. Biomed Eng Online 2016;15:115.

4. Wang DD, Gheewala N, Shah R, et al. ThreeDimensional Printing for Planning of Structural Heart Interventions. Interv Cardiol Clin 2018;7:415-23.

5. Huang Q, Zeng Z. A Review on Real-Time 3D Ultrasound Imaging Technology. Biomed Res Int 2017;2017:6027029.

6. Farooqi KM, Sengupta PP. Echocardiography and threedimensional printing: sound ideas to touch a heart. J Am Soc Echocardiogr 2015;28:398-403.

7. Kim MS, Hansgen AR, Wink O, et al. Rapid prototyping: a new tool in understanding and treating structural heart disease. Circulation 2008;117:2388-94.

8. Ryan JR, Moe TG, Richardson R, et al. A novel approach to neonatal management of tetralogy of Fallot, with pulmonary atresia, and multiple aortopulmonary collaterals. JACC Cardiovasc Imaging 2015;8:103-4.

9. Bompotis G, Meletidou M, Karakanas A, et al. Transcatheter Aortic Valve Implantation using 3-D printing modeling assistance. A single-center experience. Hellenic J Cardiol 2019. [Epub ahead of print].

10. Pellegrino PL, Fassini G, DI Biase M, et al. Left Atrial Appendage Closure Guided by 3D Printed Cardiac Reconstruction: Emerging Directions and Future Trends. J Cardiovasc Electrophysiol 2016;27:768-71.

11. Yang DH, Kang JW, Kim N, et al. Myocardial 3-Dimensional Printing for Septal Myectomy Guidance in a Patient With Obstructive Hypertrophic Cardiomyopathy. Circulation 2015;132:300-1.

12. Mashari A, Knio Z, Jeganathan J, et al. Hemodynamic Testing of Patient-Specific Mitral Valves Using a Pulse Duplicator: A Clinical Application of Three-Dimensional Printing. J Cardiothorac Vasc Anesth 2016;30:1278-85.

13. Sulkin MS, Widder E, Shao C, et al. Three-dimensional printing physiology laboratory technology. Am J Physiol 
Heart Circ Physiol 2013;305:H1569-73.

14. Biglino G, Capelli C, Wray J, et al. 3D-manufactured patient-specific models of congenital heart defects for communication in clinical practice: feasibility and acceptability. BMJ Open 2015;5:e007165.

15. Costello JP, Olivieri LJ, Su L, et al. Incorporating threedimensional printing into a simulation-based congenital heart disease and critical care training curriculum for resident physicians. Congenit Heart Dis 2015;10:185-90.

16. Mitsouras D, Liacouras P, Imanzadeh A, et al. Medical 3D Printing for the Radiologist. Radiographics 2015;35:1965-88.

17. Olivieri LJ, Krieger A, Loke YH, et al. Three-dimensional printing of intracardiac defects from three-dimensional echocardiographic images: feasibility and relative accuracy. J Am Soc Echocardiogr 2015;28:392-7.

18. Mahmood F, Owais K, Taylor C, et al. Three-dimensional printing of mitral valve using echocardiographic data. JACC Cardiovasc Imaging 2015;8:227-9.

19. Wang DD, Eng M, Kupsky D, et al. Application of 3-Dimensional Computed Tomographic Image Guidance to WATCHMAN Implantation and Impact on Early Operator Learning Curve: Single-Center Experience. JACC Cardiovasc Interv 2016;9:2329-40.

20. Greil GF, Wolf I, Kuettner A, et al. Stereolithographic reproduction of complex cardiac morphology based on high spatial resolution imaging. Clin Res Cardiol 2007;96:176-85.

21. Yoo SJ, Thabit O, Kim EK, et al. 3D printing in medicine of congenital heart diseases. 3D Print Med 2015;2:3.

22. Rengier F, Mehndiratta A, von Tengg-Kobligk H, et al. $3 \mathrm{D}$ printing based on imaging data: review of medical applications. Int J Comput Assist Radiol Surg 2010;5:335-41.

23. Bidgood WD Jr, Horii SC, Prior FW, et al. Understanding and using DICOM, the data interchange standard for biomedical imaging. J Am Med Inform Assoc 1997;4:199-212.

24. Varma DR. Managing DICOM images: Tips and tricks for the radiologist. Indian J Radiol Imaging 2012;22:4-13.

25. Goldman LW. Principles of CT: multislice CT. J Nucl Med Technol 2008;36:57-68; quiz 75-6.

26. Nasis A, Mottram PM, Cameron JD, et al. Current and evolving clinical applications of multidetector cardiac CT in assessment of structural heart disease. Radiology 2013;267:11-25

27. Ohnesorge B, Flohr T, Becker C, et al. Cardiac imaging by means of electrocardiographically gated multisection spiral CT: initial experience. Radiology 2000;217:564-71.

28. Mashari A, Montealegre-Gallegos M, Knio Z, et al. Making three-dimensional echocardiography more tangible: a workflow for three-dimensional printing with echocardiographic data. Echo Res Pract 2016;3:R57-64.

29. Vegas A. Three-dimensional transesophageal echocardiography: Principles and clinical applications. Ann Card Anaesth 2016;19:S35-43.

30. Song H, Zhou Q, Zhang L, et al. Evaluating the morphology of the left atrial appendage by a transesophageal echocardiographic 3-dimensional printed model. Medicine (Baltimore) 2017;96:e7865.

31. Leotta DF, Starnes BW. Custom fenestration templates for endovascular repair of juxtarenal aortic aneurysms. J Vasc Surg 2015;61:1637-41.

32. Byrne N, Velasco Forte M, Tandon A, et al. A systematic review of image segmentation methodology, used in the additive manufacture of patient-specific 3D printed models of the cardiovascular system. JRSM Cardiovasc Dis 2016;5:2048004016645467.

33. Schievano S, Taylor AM, Capelli C, et al. First-in-man implantation of a novel percutaneous valve: a new approach to medical device development. EuroIntervention 2010;5:745-50.

34. Wang DD, Eng M, Greenbaum A, et al. Predicting LVOT Obstruction After TMVR. JACC Cardiovasc Imaging 2016;9:1349-52.

35. Fan S, Chan A, Au S, et al. Personalised anaesthesia: threedimensional printing of facial prosthetic for facial deformity with difficult airway. Br J Anaesth 2018;121:675-8.

36. Giannopoulos AA, Mitsouras D, Yoo SJ, et al. Applications of $3 \mathrm{D}$ printing in cardiovascular diseases. Nat Rev Cardiol 2016;13:701-18.

37. Fan Y, Yang F, Cheung GS, et al. Device Sizing Guided by Echocardiography-Based Three-Dimensional Printing Is Associated with Superior Outcome after Percutaneous Left Atrial Appendage Occlusion. J Am Soc Echocardiogr 2019;32:708-719.e1.

38. Chung P, Heller JA, Etemadi M, et al. Rapid and low-cost prototyping of medical devices using 3D printed molds for liquid injection molding. J Vis Exp 2014;(88):e51745.

39. Palacios IF, Arzamendi D. Structural heart intervention. Beyond transcatheter valve therapy. Rev Esp Cardiol (Engl Ed) 2012;65:405-13.

40. Fan Y, Kwok KW, Zhang Y, et al. Three-Dimensional Printing for Planning Occlusion Procedure for a DoubleLobed Left Atrial Appendage. Circ Cardiovasc Interv 2016;9:e003561.

41. Garekar S, Bharati A, Chokhandre M, et al. Clinical Application and Multidisciplinary Assessment of Three Dimensional Printing in Double Outlet Right Ventricle 
With Remote Ventricular Septal Defect. World J Pediatr Congenit Heart Surg 2016;7:344-50.

42. Gosnell J, Pietila T, Samuel BP, et al. Integration of Computed Tomography and Three-Dimensional Echocardiography for Hybrid Three-Dimensional Printing in Congenital Heart Disease. J Digit Imaging 2016;29:665-9.

43. Ripley B, Kelil T, Cheezum MK, et al. 3D printing based on cardiac CT assists anatomic visualization prior to transcatheter aortic valve replacement. J Cardiovasc Comput Tomogr 2016;10:28-36.

44. So KC, Fan Y, Sze L, et al. Using Multimaterial 3-Dimensional Printing for Personalized Planning of Complex Structural Heart Disease Intervention. JACC Cardiovasc Interv 2017;10:e97-8.

45. Hell MM, Achenbach S, Yoo IS, et al. 3D printing for sizing left atrial appendage closure device: headto-head comparison with computed tomography and transoesophageal echocardiography. EuroIntervention 2017;13:1234-41.

46. Liu P, Liu R, Zhang Y, et al. The Value of 3D Printing Models of Left Atrial Appendage Using Real-Time 3D Transesophageal Echocardiographic Data in Left Atrial Appendage Occlusion: Applications toward an Era of Truly Personalized Medicine. Cardiology 2016;135:255-61.

47. Robinson SS, Alaie S, Sidoti H, et al. Patient-specific design of a soft occluder for the left atrial appendage. Nat Biomed Eng 2018;2:8-16.

48. Obasare E, Mainigi SK, Morris DL, et al. CT based 3D printing is superior to transesophageal echocardiography for pre-procedure planning in left atrial appendage device closure. Int J Cardiovasc Imaging 2018;34:821-31.

49. Jung JI, Koh YS, Chang K. 3D Printing Model before and after Transcatheter Aortic Valve Implantation for a Better Understanding of the Anatomy of Aortic Root. Korean Circ J 2016;46:588-9.

50. Qian Z, Wang K, Liu S, et al. Quantitative Prediction of Paravalvular Leak in Transcatheter Aortic Valve Replacement Based on Tissue-Mimicking 3D Printing. JACC Cardiovasc Imaging 2017;10:719-31.

51. Hosny A, Dilley JD, Kelil T, et al. Pre-procedural fittesting of TAVR valves using parametric modeling and 3D printing. J Cardiovasc Comput Tomogr 2019;13:21-30.

52. Maragiannis D, Jackson MS, Igo SR, et al. Replicating Patient-Specific Severe Aortic Valve Stenosis With Functional 3D Modeling. Circ Cardiovasc Imaging 2015;8:e003626.

53. Binder TM, Moertl D, Mundigler G, et al. Stereolithographic biomodeling to create tangible hard copies of cardiac structures from echocardiographic data: in vitro and in vivo validation. J Am Coll Cardiol 2000;35:230-7.

54. Theodoropoulos KC, Papachristidis A, Fonseca T, et al. Understanding the anatomy of a perforated mitral valve: From 2D echocardiography to 3D printing. Hellenic J Cardiol 2018. [Epub ahead of print].

55. Mahmood F, Owais K, Montealegre-Gallegos M, et al. Echocardiography derived three-dimensional printing of normal and abnormal mitral annuli. Ann Card Anaesth 2014;17:279-83.

56. Little SH, Vukicevic M, Avenatti E, et al. 3D Printed Modeling for Patient-Specific Mitral Valve Intervention: Repair With a Clip and a Plug. JACC Cardiovasc Interv 2016;9:973-5.

57. Dankowski R, Baszko A, Sutherland M, et al. 3D heart model printing for preparation of percutaneous structural interventions: description of the technology and case report. Kardiol Pol 2014;72:546-51.

58. Chaowu Y, Hua L, Xin S. Three-Dimensional Printing as an Aid in Transcatheter Closure of Secundum Atrial Septal Defect With Rim Deficiency: In Vitro Trial Occlusion Based on a Personalized Heart Model. Circulation 2016;133:e608-10.

59. Bartel T, Rivard A, Jimenez A, et al. Three-dimensional printing for quality management in device closure of interatrial communications. Eur Heart J Cardiovasc Imaging 2016;17:1069.

60. Thakkar AN, Chinnadurai P, Breinholt JP, et al. Transcatheter closure of a sinus venosus atrial septal defect using $3 \mathrm{D}$ printing and image fusion guidance. Catheter Cardiovasc Interv 2018;92:353-7.

61. Wang Z, Liu Y, Xu Y, et al. Three-dimensional printingguided percutaneous transcatheter closure of secundum atrial septal defect with rim deficiency: First-in-human series. Cardiol J 2016;23:599-603.

62. Holmes DR, Reddy VY, Turi ZG, et al. Percutaneous closure of the left atrial appendage versus warfarin therapy for prevention of stroke in patients with atrial fibrillation: a randomised non-inferiority trial. Lancet 2009;374:534-42.

63. Reddy VY, Gibson DN, Kar S, et al. Post-Approval U.S. Experience With Left Atrial Appendage Closure for Stroke Prevention in Atrial Fibrillation. J Am Coll Cardiol 2017;69:253-61.

64. Reddy VY, Holmes D, Doshi SK, et al. Safety of percutaneous left atrial appendage closure: results from the Watchman Left Atrial Appendage System for Embolic Protection in Patients with AF (PROTECT AF) clinical trial and the Continued Access Registry. Circulation 
2011;123:417-24.

65. Viles-Gonzalez JF, Kar S, Douglas P, et al. The clinical impact of incomplete left atrial appendage closure with the Watchman Device in patients with atrial fibrillation: a PROTECT AF (Percutaneous Closure of the Left Atrial Appendage Versus Warfarin Therapy for Prevention of Stroke in Patients With Atrial Fibrillation) substudy. J Am Coll Cardiol 2012;59:923-9.

66. Masoudi FA, Calkins H, Kavinsky CJ, et al. 2015 ACC/ HRS/SCAI Left Atrial Appendage Occlusion Device Societal Overview: A professional societal overview from the American College of Cardiology, Heart Rhythm Society, and Society for Cardiovascular Angiography and Interventions. Catheter Cardiovasc Interv 2015;86:791-807.

67. Otton JM, Spina R, Sulas R, et al. Left Atrial Appendage Closure Guided by Personalized 3D-Printed Cardiac Reconstruction. JACC Cardiovasc Interv 2015;8:1004-6.

68. Nishimura RA, Otto CM, Bonow RO, et al. 2017 AHA/ ACC Focused Update of the 2014 AHA/ACC Guideline for the Management of Patients With Valvular Heart Disease: A Report of the American College of Cardiology/ American Heart Association Task Force on Clinical Practice Guidelines. J Am Coll Cardiol 2017;70:252-89.

69. Moat NE. Will TAVR Become the Predominant Method for Treating Severe Aortic Stenosis? N Engl J Med 2016;374:1682-3.

70. Smith CR, Leon MB, Mack MJ, et al. Transcatheter versus surgical aortic-valve replacement in high-risk patients. $\mathrm{N}$ Engl J Med 2011;364:2187-98.

71. Carabello BA. Transcatheter aortic-valve implantation for aortic stenosis in patients who cannot undergo surgery. Curr Cardiol Rep 2011;13:173-4.

72. Figulla HR, Webb JG, Lauten A, et al. The transcatheter valve technology pipeline for treatment of adult valvular heart disease. Eur Heart J 2016;37:2226-39.

73. Schmauss D, Schmitz C, Bigdeli AK, et al. Threedimensional printing of models for preoperative planning and simulation of transcatheter valve replacement. Ann Thorac Surg 2012;93:e31-3.

74. Gallo M, D'Onofrio A, Tarantini G, et al. 3D-printing model for complex aortic transcatheter valve treatment. Int J Cardiol 2016;210:139-40.

75. Lee AP, Leong CW, Kwok KW, et al. Using 3D Printed Models for Planning Transcatheter Aortic Valve Implantation in Patients with Bicuspid Aortic Valve. J Am Coll Cardiol 2018;71:abstr 1130.

76. Lee PW, Leong CW, Fan Y, et al. Avoiding coronary occlusion in TAVI: the role of procedural simulation with patient-specific 3D-printed aortic root models. PCR Tokyo Valves 2019. abstr Tokyo19A-OP013.

77. Nkomo VT, Gardin JM, Skelton TN, et al. Burden of valvular heart diseases: a population-based study. Lancet 2006;368:1005-11.

78. Iung B, Vahanian A. Epidemiology of valvular heart disease in the adult. Nat Rev Cardiol 2011;8:162-72.

79. Eggebrecht H, Mehta RH, Lubos E, et al. MitraClip in High- Versus Low-Volume Centers: An Analysis From the German TRAMI Registry. JACC Cardiovasc Interv 2018;11:320-2.

80. Webb JG, Murdoch DJ, Boone RH, et al. Percutaneous Transcatheter Mitral Valve Replacement: First-in-Human Experience With a New Transseptal System. J Am Coll Cardiol 2019;73:1239-46.

81. Vukicevic M, Puperi DS, Jane Grande-Allen K, et al. 3D Printed Modeling of the Mitral Valve for Catheter-Based Structural Interventions. Ann Biomed Eng 2017;45:508-19.

82. Vaquerizo B, Theriault-Lauzier P, Piazza N. Percutaneous Transcatheter Mitral Valve Replacement: Patient-specific Three-dimensional Computer-based Heart Model and Prototyping. Rev Esp Cardiol (Engl Ed) 2015;68:1165-73.

83. El Sabbagh A, Eleid M, Said S, et al. 3D printing for procedural simulation of transcatheter mitral valve replacement in patients with mitral annular calcification. J Am Coll Cardiol 2017;69:1142.

84. Price MJ, Smith MR, Rubenson DS. Utility of on-line three-dimensional transesophageal echocardiography during percutaneous atrial septal defect closure. Catheter Cardiovasc Interv 2010;75:570-7.

85. Lee AP, Lam YY, Yip GW, et al. Role of real time threedimensional transesophageal echocardiography in guidance of interventional procedures in cardiology. Heart 2010;96:1485-93.

86. Schrot J, Pietila T, Sahu A. State of the art: 3D printing for creating compliant patient-specific congenital heart defect models. J Cardiovasc Magn Reson 2014;16:W19.

87. Song H, Zhou Q, Deng Q, et al. Morphologic Assessment of the Left Atrial Appendage in Patients with Atrial Fibrillation by Gray Values-Inverted Volume-Rendered Imaging of Three-Dimensional Transesophageal Echocardiography: A Comparative Study with Computed Tomography. J Am Soc Echocardiogr 2016;29:1100-8.

88. Budge LP, Shaffer KM, Moorman JR, et al. Analysis of in vivo left atrial appendage morphology in patients with atrial fibrillation: a direct comparison of transesophageal echocardiography, planar cardiac CT, and segmented three-dimensional cardiac CT. J Interv Card 


\section{Page 13 of 13}

Electrophysiol 2008;23:87-93.

89. Jilaihawi H, Doctor N, Kashif M, et al. Aortic annular sizing for transcatheter aortic valve replacement using cross-sectional 3-dimensional transesophageal echocardiography. J Am Coll Cardiol 2013;61:908-16.

90. Wunderlich NC, Beigel R, Swaans MJ, et al. Percutaneous interventions for left atrial appendage

Cite this article as: Fan Y, Wong RH, Lee AP. Threedimensional printing in structural heart disease and intervention. Ann Transl Med 2019;7(20):579. doi: 10.21037/ atm.2019.09.73 exclusion: options, assessment, and imaging using 2D and $3 \mathrm{D}$ echocardiography. JACC Cardiovasc Imaging 2015;8:472-88.

91. Li H, Qingyao, Bingshen, et al. Application of 3D printing technology to left atrial appendage occlusion. Int J Cardiol 2017;231:258-63. 